\title{
Estresse, ansiedade e qualidade de vida em professores: efeitos do relaxamento progressivo
}

\author{
Geisa Rodrigues de Freitas', https://orcid.org/0000-0001-8506-3833 \\ Sandra Leal Calais', https://orcid.org/0000-0001-5704-4643
}

Hugo Ferrari Cardoso', https://orcid.org/0000-0003-1960-2936

\begin{abstract}
Resumo
Estudos indicam presença de sintomas de estresse, ansiedade e baixa qualidade de vida ligada à docência no ensino público; entretanto, poucos são de caráter interventivo. O objetivo desta pesquisa foi investigar os efeitos do Relaxamento Progressivo de Jacobson adaptado por Sandor nas medidas de estresse, ansiedade e qualidade de vida (QV) de professores de escolas públicas. Constituíram a amostra 105 professores de escolas públicas estaduais, divididos em três grupos: intervenção, palestra e passagem de tempo. Utilizou-se comparação pré e pós-teste com os instrumentos ISSL, EVENT, WHOQOL, BAI. No grupo experimental, identificou-se redução significativa $(p=0,004)$ no pós-teste para estresse,e alterações na percepção do estresse no trabalho, ansiedade e QV, sem significância estatística, entretanto, indicando tendência de melhora nos sintomas. Verificou-se que esse relaxamento foi favorável aos professores. Sugerem-se outras investigações de caráter interventivo com docentes, possibilitando-se a ampliação do repertório comportamental de respostas saudáveis frente a estímulos ambientais aversivos.
\end{abstract}

Palavras-chave: Estresse; ansiedade; terapia de relaxamento.

\section{Stress, anxiety and quality of life in teachers: effects of progressive relaxation}

\begin{abstract}
Studies indicate the presence of symptoms of stress, anxiety and low quality of life linked to teaching in public education; however, few are of an interventional nature. The objective of this research was to investigate the effects of Jacobson's Progressive Relaxation on the stress, anxiety, and quality of life (QOL) measures of public school teachers. The sample consisted of 105 teachers from state public schools, divided into three groups: intervention, lecture, and passage of time. Pre- and post-test comparison has used with ISSL, EVENT, WHOQOL, BAI instruments. In the experimental group, a significant reduction $(p=0.004)$ has observed in the post-test for stress, and changes in the perception of work stress, anxiety and QoL, without statistical significance, however, indicating a tendency to improve symptoms. It was found that this relaxation was favorable to teachers. Other investigations of an interventional nature with teachers has suggested, making possible the amplification of the behavioral repertoire of healthy responses to aversive environmental stimuli.
\end{abstract}

Keywords: Stress; anxiety; relaxation therapy.

\section{Estrés, ansiedad y calidad de vida en profesores: efectos del relajamiento progresivo}

\section{Resumen}

Estudios indican presencia de síntomas de estrés, ansiedad y baja calidad de vida relacionada a la docencia en la enseñanza pública; sin embargo, pocos son de carácter de intervención. El objetivo de esta investigación fue averiguar los efectos del Relajamiento Progresivo de Jacobson adaptado por Sandor en las medidas de estrés, ansiedad y calidad de vida (QV) de profesores de escuelas públicas. Constituyeron la muestra 105 profesores de escuelas públicas estaduales, divididos en tres grupos: intervención, ponencia y pasaje de tiempo. Se utilizó comparación antes y tras test con los instrumentos ISSL, EVENT, WHOQOL, BAI. En el grupo experimental, se identificó reducción significativa $(p=0,004)$ en el post-test para estrés, y alteraciones en la percepción del estrés en el trabajo, ansiedad y QV, sin significancia estadística, sin embargo, indicando tendencia de mejora en los síntomas. Se verificó que este relajamiento fue favorable a los profesores. Se sugiere otras investigaciones de carácter de intervención con docentes, posibilitándose la ampliación del repertorio comportamental de respuestas saludables frente a estímulos ambientales aversivos.

Palabras clave: Estrés; ansiedad; terapia de relajamiento.

1 Universidade Estadual Paulista Júlio de Mesquita Filho, Bauru - São Paulo - Brasil; geisa.rodrigues@ymail.com; scalais@fc.unesp.br; hfcardoso@fc.unesp.br 


\section{Introdução}

O endocrinologista Hans Hugo Bruno Selye (19071982) utilizou o termo estresse para denominar as forças que qualquer organismo tem para manter-se e adaptar-se. Propôs a denominação da Síndrome de Adaptação Geral (SAG) ao estresse constituída por três estágios: reação de alarme, fase de resistência e fase de esgotamento (Selye, 1950). Lipp (1984) complementa que estresse é uma reação psicológica, com componentes emocionais, físicos, mentais e químicos, frente a estímulos que irritam, amedrontam, excitam e/ou confundem a pessoa. Juntamente com Malagris (Lipp \& Malagris, 2001), Lipp propôs a fase de quase-exaustão, acrescentando-a ao modelo de Selye, sugerindo uma classificação sintomatológica quadrifásica. Essa fase antecipa-se à de exaustão ou esgotamento, e caracteriza-se pela condição em que o organismo não consegue manter sua homeostase e nem lutar contra o estressor; a pessoa debilita-se mais e surgem doenças, apesar de o indivíduo ainda realizar suas atividades cotidianas.

Algumas dessas debilidades físicas são citadas por Chep (2013) como úlceras estomacais, aumento na pressão do sangue, perda de sono e possíveis problemas articulares decorrentes. Gebel (2012) comenta que o estresse pode favorecer o surgimento de diabetes tipo 2, e que o estresse crônico, mesmo que em baixo nível, afeta o cérebro, coração, pulmões, músculos e tem sido associado a vários problemas de saúde. Vê-se como alarmante o fato de que estudos científicos realizados com professores indicam a presença de sintomas de estresse, ansiedade e/ou baixa Qualidade de Vida (QV) nessa população (Andrade \& Cardoso, 2012; Brum \& cols., 2012; Colares, Lopes, Barbosa Filho, Bavaresco, \& Scherer, 2015; Moy \& cols., 2014).

Naghieh, Montgomery, Bonell, Thompson e Aber (2015) referem que a profissão dos docentes tem alta prevalência de estresse, podendo afetar negativamente sua saúde, bem-estar e ter impacto na educação das crianças e no orçamento público, devido ao número de licenças médicas e da rotatividade. Simplício e Andrade (2011) realizaram estudo com 34 professores da rede municipal para verificar a relação entre saúde e trabalho. Foi feita análise referente a quatro momentos da trajetória dos docentes: estágio, início de carreira, experiência e final de carreira. Em cada etapa, três categorias surgiram como sintomas prejudiciais à saúde: exaustão emocional, despersonalização, diminuição da realização pessoal no trabalho; todos fatores relacionados ao estresse. Considerando dados como esses, verifica-se a importância de também se buscar a identificação das possíveis fontes estressoras relacionadas. Em amostra de 404 professores de escolas públicas, Vale, Maciel e Carlotto (2015) aplicaram a Escala de Percepção de Estressores Ocupacionais dos Professores e obtiveram que o fator "relações com alunos e responsáveis" foi o que os professores selecionaram como o mais estressor. Em segundo lugar, ficou "carga de trabalho", seguido pelos fatores "aspectos físicos e ambientais do trabalho", "crescimento na carreira" e "capacitação profissional".
A ocorrência da condição de estresse pode vir acompanhada de outros sintomas, como os de ansiedade (Andrade \& Cardoso, 2012; Apóstolo \& cols., 2011). A presença de ansiedade também tem sido identificada em pesquisas com docentes. Silveira, Enumo e Batista (2014) realizaram um estudo descritivo e correlacional com 21 professores de salas multisseriadas brasileiras utilizando questionário sociodemográfico, Inventário de Sintomas de Stress para adultos de Lipp (ISSL), Inventário de Ansiedade de Beck (BAI) e Escala de Modos de Enfrentamento de Problemas de Seidl, Tróccoli e Zannon (EMEP). Relataram que 30\% da amostra apresentaram sintomas de ansiedade em grau moderado ou grave, e encontraram correlação positiva entre estresse e ansiedade. Ressalvaram, entretanto, não ter sido possível estabelecer como se dá a relação entre estas variáveis, nem qualificar a existência de uma relação causal entre ambas.

Almeida e cols. (2014) investigaram, em uma amostra de 93 participantes, a relação entre características vocais e emocionais de professores que apresentaram alta e baixa ansiedade. Os grupos identificados como de alta ansiedade foram os com mais sintomas indicativos de estresse e depressão e maior número de comprometimentos vocais. Concluíram que as pessoas com alta ansiedade, em especial os professores, apresentaram maior comprometimento emocional, vocal e na QV.

Apesar do caráter multifacetado do construto ansiedade (DeSousa \& cols., 2013) esta pode ser definida como uma condição orientada para o futuro, caracterizada pela apreensão relativa à percepção de não poder controlar ou prever eventos potencialmente aversivos; a existência de sintomas corporais de tensão física; e o desvio do foco de atenção para esses eventos potencialmente aversivos ou às respostas afetivas eliciadas por eles (Barlow \& Durand, 2008). A recorrência de exposição frente a estímulos estressores pode eliciar respostas antecipatórias aversivas, havendo, portanto, a relação entre ansiedade e estresse.

Dada a presença marcante de sintomas de ansiedade e estresse identificados na população docente, e sabendo-se que o conceito de QV corresponde à percepção do indivíduo de sua posição na vida, em seu contexto da cultura e sistema de valores e, em relação aos seus objetivos, expectativas, padrões e preocupações (Fleck \& cols., 2000), estudos têm investigado como a medida de QV tem sido afetada nessa população, em seus diferentes domínios. A QV se refere, portanto, a uma avaliação subjetiva e influenciada pelos contextos cultural, social e ambiental, ultrapassando o conceito de bem-estar, estado de saúde ou mental (Chachamovich \& Fleck, 2008).

Colares e cols. (2015) realizaram uma revisão sistemática das publicações científicas sobre os indicadores de QV de professores em cinco bases de dados, de janeiro de 1990 a agosto de 2013. Sobre os resultados obtidos, relataram que a maioria das publicações indicou insatisfação dos professores com relação à estrutura física, remuneração, condições organizacionais. Dos estudos em que o WHOQOL foi utilizado como instrumento, a maioria das médias obtidas para QV foi abaixo dos 70 pontos, excetuando-se o domínio 
Relações Sociais; sendo os piores: Meio Ambiente e Físico. Damásio, Melo e Silva (2013) pesquisaram os índices de QV (WHOQOL-bref), sentido de vida e bem-estar psicológico em 517 professores de escolas públicas e privadas brasileiras. Verificou o número significativo de profissionais com índices negativos de QV, sentido de vida e bem-estar psicológico.

Tabeleão, Tomasi e Neves (2011) realizaram um estudo com 601 professores de escolas estaduais e municipais para investigar os níveis de QV destes profissionais e de relacioná-los às características sociodemográficas da amostra, utilizando o WHOQOL abreviado. Os índices médios obtidos para os diferentes domínios do instrumento foram: 69,2 para Físico; 70,6 para Psicológico; 72,5 para Relações Sociais e 60,7 para Ambiente. A maioria dos profissionais encontrados eram mulheres com mais de 20 anos de docência. Caye, Ulbricht e Neves (2014) pesquisaram a qualidade de vida no trabalho de 55 professores de português e matemática de Ensino Fundamental em escolas públicas e privadas utilizando questionário sociodemográfico e o WHOQOL-bref. Indicaram que a maior parte dos professores (76\%) classificou positivamente a sua QV. Na análise por domínios, obtiveram: Relações Sociais com a melhor média (73,31), Físico $(71,16)$, Psicológico $(70,81)$ seguido pelo Meio Ambiente, que mais impactou negativamente na qualidade de vida dos professores com apenas 57,31 pontos.

Nota-se significativa quantidade de levantamentos científicos sobre a carreira docente e seus aspectos estressores, ansiogênicos e sua relativa reflexão na avaliação da QV desses profissionais. Andrade e Cardoso (2012), em uma revisão de literatura sobre a Síndrome de Burnout em docentes, informam ter encontrado estudos predominantemente descritivos sobre o assunto e indicam a falta de programas de prevenção e de intervenção para cuidar do estresse do professor. Alguns autores chamam a atenção para a importância de políticas públicas que visem promover a saúde do professor, e relatam que a maioria das intervenções voltadas ao bem-estar de docentes têm sido realizadas individualmente. Nesse sentido, podem não atingir, de forma efetiva, os sintomas de estresse desencadeados no local de trabalho. Sugerem que as intervenções realizadas em nível organizacional sejam um potencial para alcançarem uma melhora mais efetiva do estresse desses profissionais (Carlotto, Dias, Batista, \& Dihel, 2015; Damásio \& cols., 2013; Naghieh \& cols., 2015; Simplício \& Andrade, 2011). Foi voltado a essa lacuna que, no presente estudo, buscou-se investigar uma proposta interventiva visando redução de sintomas de estresse e ansiedade e melhora da avaliação de QV. Neste sentido, dentre as propostas interventivas existentes, encontram-se as técnicas de relaxamento que visam promover estados opostos ao efeito negativo de situações de ativação do corpo causadas por estímulos estressores (Jacobson,1976).

O relaxamento muscular progressivo proposto pelo médico Edmund Jacobson (1888-1983) apresenta-se como comportamento concorrente ao do estresse por eliciar respostas orgânicas opostas às de tensão fisiológica provocada por situações estressoras, como regulação da pressão san- guínea, frequência cardíaca, respiração, circulação, reflexo pupilar e trabalho muscular. A partir do momento em que se pode controlar, diretamente, os músculos, mas não o sistema nervoso autônomo, as consequências são de longo alcance. Transitar do estado de tensão para o de relaxamento dentro do prazo de poucos minutos ou menos é resultado da insistência reiterada na prática do controle da tensão, sendo que alcançar esse hábito deveria ser um aprendizado mais fácil do que ocorre. Para concluir o curso desse aprendizado e relaxar logo que a tensão apareça, torna-se necessário treino de dias, meses ou até anos para muitas pessoas e o objetivo não é encerrar as tensões, pois fazem parte da vida, mas sim de utilizar o relaxamento como meio para controlá-las (Jacobson, 1976).

A pessoa deve aprender o relaxamento muscular a partir da prática regular do trabalho de grupos musculares diferentes, sendo estes divididos em fases didáticas conforme a região do corpo: braços, pernas, tronco, pescoço, testa, cenho, olhos, imaginação visual, maçãs do rosto, maxilares, lábios, língua, fala e discurso imaginário (Jacobson, 1976). Considerando que esses passos exigem intervalos prolongados de treino em cada grupo muscular, Sandor (1974) propôs uma adaptação do método a partir da utilização de seis passos, cada qual trabalhando determinado grupo muscular.

Esgalha (2010) pesquisou o uso do relaxamento muscular progressivo de Jacobson e sua eficácia na alteração das medidas de estresse (ISSL) e ansiedade (BAI) em pessoas com dor crônica devido à Disfunção da Articulação Temporomandibular. Verificou redução de estresse, porém não houve alterações significativas em ansiedade para o grupo experimental. Lopes, Santos e Lopes (2008) verificaram os efeitos da mesma técnica no controle da ansiedade e desesperança em 30 mulheres diagnosticadas com câncer. Identificaram redução das medidas obtidas para ansiedade imediatamente após a aplicação da técnica, porém, esta medida não se manteve ao longo do tempo. Não há relatos, no entanto, de intervenções com professores.

Assim, o presente estudo visou investigar maneiras de instrumentalizar os docentes para que possam apresentar, frente a estímulos aversivos, comportamentos mais favoráveis à $\mathrm{QV}$, redução de sintomas de estresse e ansiedade. Utilizou-se a técnica do Relaxamento Progressivo de Jacobson adaptado por Sandor (1974) com o objetivo de verificar seus efeitos nas medidas de estresse, ansiedade e QV de professores de escolas públicas estaduais.

\section{Método}

\section{Participantes}

Constituíram a amostra desta pesquisa 105 professores de escolas públicas estaduais em exercício. Foram critérios de exclusão: presença de diagnósticos psiquiátricos, gravidez, e tempo de função inferior a um ano. 
O grupo experimental (G1) constituiu-se de 39 professores que tiveram interesse e disponibilidade de participar de seis encontros para treino de Relaxamento Muscular Progressivo. O grupo de palestra (G2) constituiu-se de 33 professores que participaram de uma palestra sobre estresse; o grupo controle (G3), por 33 professores que apenas preencheram os instrumentos, sem terem participado de nenhuma intervenção ou palestra. $O$ delineamento foi de trabalho interventivo com comparação pré e pós-teste entre os diferentes grupos a fim de permitir investigar a evolução das médias grupais frente a três diferentes tipos de intervenção.

\section{Instrumentos}

Foram utilizados: questionário sociodemográfico a fim de coletar os dados pessoais e profissionais dos participantes; o Inventário de Sintomas de Stress para Adultos de Lipp (ISSL) (Lipp, 2000) para identificar a presença de estresse, a fase de estresse em que a pessoa se encontrava e a predominância de sintomas (físicos e/ou psicológicos). A Escala de Vulnerabilidade ao Estresse no Trabalho (EVENT) (Sisto, Noronha, Santos, \& Baptista, 2007) visou avaliação da vulnerabilidade do professor frente aos estressores identificados no ambiente do trabalho. O Inventário de Ansiedade de Beck (BAI) (Cunha, 2001) é constituído por 21 itens relativos a sintomas de ansiedade e foi utilizado para verificar quantidade de sintomas apresentados pelo indivíduo e seu respectivo grau de intensidade, podendo ser identificado como: ansiedade mínima, leve, moderada ou grave. O Questionário de Qualidade de Vida WHOQOL Abreviado (Chachamovich\&Fleck, 2008) visou estimar a QV dos participantes.

\section{Procedimento}

Todos os cuidados éticos foram tomados, conforme a Resolução n 466/12 do Conselho Nacional de Saúde, sob parecer $n^{\circ} 430.900$. Os professores interessados em participar da pesquisa receberam o Termo de Consentimento Livre e Esclarecido e foram informados quanto à pesquisa. A escolha de qual professor iria participar de cada grupo decorreu: do interesse do docente, da disponibilidade da escola para permitir a realização dos encontros de treino do relaxamento (as escolas foram escolhidas por conveniência), dos horários de ATPC (Aula de Trabalho Pedagógico Coletivo) em que o professor estaria escalado, bem como dos critérios de exclusão definidos. A coleta de dados e aplicação do relaxamento foi realizada nas próprias escolas, sendo que, no caso do relaxamento (G1), os colchonetes e travesseiros foram levados pela pesquisadora às escolas em cada aplicação.

Os três grupos (G1, G2 e G3) foram submetidos à condição inicial de responder aos instrumentos (pré-teste). Em seguida, o G1 participou de seis encontros de intervenção com o relaxamento muscular progressivo, seguindo roteiro pré-determinado tendo sido focados na primeira sessão, braços; na segunda, pernas; na terceira, abdômen e pescoço; na quarta, testa e cenho; na quinta, olhos e imaginação visual e na sexta, órgãos da linguagem. O participante, inicialmente, recebia as informações sobre o relaxamento e algumas dúvidas sobre as sessões ou as execuções dos exercícios eram esclarecidas. Também era informado que utilizasse a técnica em situações do cotidiano. Ao G2, foi oferecida uma palestra informativa sobre estresse de duração média de 40 minutos, com explicações teóricas sobre estratégias de enfrentamento de situações estressoras. O G3 foi caracterizado pela espera da passagem de tempo. Após as intervenções, os participantes dos três grupos responderam novamente aos instrumentos iniciais (pós-teste).

\section{Resultados}

Foi realizada a comparação intragrupos e intergrupos por meio de tratamentos estatísticos dos dados avaliativos dos participantes dos Grupos 1, 2 e 3. As informações coletadas foram sintetizadas, quantitativamente, por meio das funções da estatística descritiva. Para comparação entre grupos, utilizou-se o método de análise de variâncias, o teste ANOVA para análise inferencial e comparação entre mais de duas médias. O nível de significância adotado neste estudo foi de $p \leq 0,05$.

$\mathrm{Na}$ caracterização da amostra, $75,2 \%$ eram do sexo feminino; $61,7 \%$ eram casados; $72,0 \%$ com filhos; $69,4 \%$ com mais de 10 anos de docência, sendo a média de 14,8 anos de exercício da profissão e o desvio padrão de 8,2 anos. A média obtida para a idade dos participantes foi de 40,8 anos e o desvio padrão foi de 9,6 anos.

Considerando a amostra total no pré-teste, de acordo com os dados obtidos na avaliação dos sintomas de estresse por meio do ISSL, verificou-se que 62\% apresentavam estresse (sendo 76,9\% na fase de resistência, 15,3\% em quase-exaustão, 6,1\% em alerta; e uma pessoa em exaustão). No pós-teste, $59 \%$ apresentaram (sendo $88,8 \%$ na fase de resistência, 6,4\% em quase-exaustão, 1,9\% em alerta; e uma pessoa em exaustão). A média obtida no pré-teste pelo ISSL mostrou equivalência na amostra da pesquisa. As médias obtidas pelo ISSL no pré e pós-teste foram, respectivamente, 12,64 e 8,28 para o G1; 13,88 e 13,31 para o G2 e 14,76 e 15,21 para o G3.Os dados pós-teste indicaram diferença estatisticamente significativa $(p=0,004)$ para o $\mathrm{G} 1$, o qual apresentou redução dos níveis de estresse.

Na EVENT, comparando-se as médias obtidas para os três fatores (Fator 1 - Clima e Funcionamento Organizacional; Fator 2 - Pressão no Trabalho e Fator 3 - Infraestrutura e Rotina e para Estresse total), verificou-se que a amostra foi equivalente frente a estas medidas. As médias do G2 e do G3 foram classificadas em Inferior para a percepção de estresse nos três fatores. Para G1, os fatores 1 e 3 foram classificados como Médio Inferiores na medida pré-teste. Foi identificada, portanto, diminuição na percepção do estresse para os três fatores, com diferença da média entre pré e 


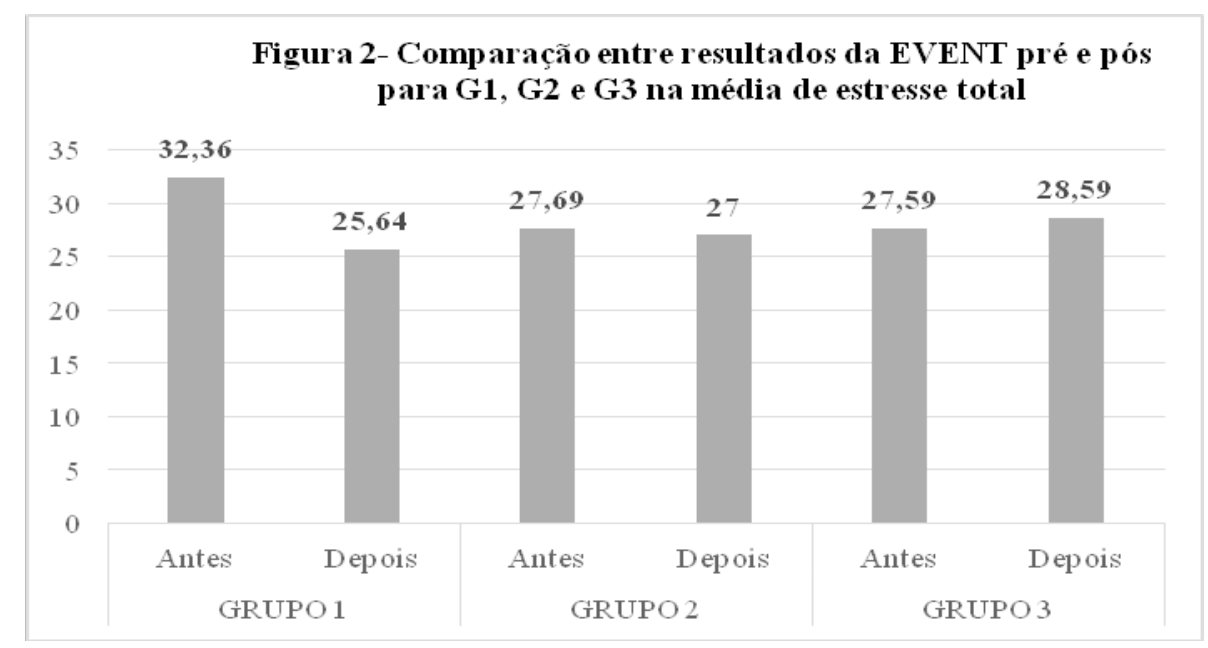

Figura 1. Comparação entre resultados pré e pós da média de estresse total da EVENT para os três grupos.

pós-teste para estresse total no valor de 6,72 , sendo que para $\mathrm{G} 2$, houve redução de 0,69 na percepção do estresse e para o G3 houve aumento do estresse percebido no valor de 1 ponto bruto nesta percepção. Na Figura 1 destaca-se a comparação entre medidas brutas pré e pós-teste da EVENT para G1, G2 e G3 frente ao estresse total.

Do total de participantes do $\mathrm{G} 1,71,8 \%$ obtiveram redução na percepção do estresse para Estresse Total, enquanto que para o $\mathrm{G} 2$, foram $45,4 \%$ e para o $\mathrm{G} 3$ foram $36,3 \%$. Considerando a análise inferencial, comparando-se as médias dos três grupos para pré e pós, a diferença obtida para o G1 não foi estatisticamente significativa, $\operatorname{com} p=0,71$.

Quanto à ansiedade, de acordo com os dados obtidos pelo BAI, do total da amostra na coleta pré-teste, $94,2 \%$ apresentaram algum grau de ansiedade, sendo que destes, $56,5 \%$ apresentaram grau Mínimo; $23,2 \%$ Leve; $16,1 \%$ Moderado e $9 \%$ Grave. A média obtida no nos pré e pós-testes para os três grupos foi, respectivamente, 13,10 e 8,38 para o G1; 12,50 e 12,44 para o G2; 13,00 e 14,03 para o G3; havendo, portanto, manutenção das médias no G2; aumento nos relatos para o $\mathrm{G} 3$ e diminuição dos sintomas relatados pelos participantes no $\mathrm{G} 1 \mathrm{com} p=0,068$.

A média geral da QV obtida pelo WHOQOL Abreviado foi de 64,17 em uma escala que vai de zero a 100 . O domínio com pior avaliação foi o Ambiente, com média de 60,20; seguido pelos domínios: Psicológico $(64,29)$, Relações Sociais $(65,93)$ e Físico $(66,14)$. Comparando-se as médias pré-teste e pós-teste para os três grupos, não foi verificada diferença estatisticamente significativa para o $\mathrm{G} 1$, tendo sido o domínio Psicológico o de maior alteração pós-intervenção com $p=0,09$.

\section{Discussão}

A identificação de altos níveis de estresse, ansiedade e reduzida $Q V$ de professores está presente em diferentes estudos científicos (Almeida \& cols., 2014; Amorim Neto,
2015; Brum \& cols., 2012; Colares, \& cols., 2015; Cunha, 2001; Damásio \& cols., 2013; Moy \& cols., 2014; Naghieh \& cols., 2015; Simplício \& Andrade, 2011; Tabeleão \& cols., 2011; Vale \& cols., 2015), mas o reduzido número de trabaIhos interventivos com a proposta de verificar a influência sobre essas variáveis (Andrade \& Cardoso, 2012) denota a importância e urgência da necessidade de trabalhos preventivos e/ou de tratamento destinados aos professores, como meio de amenizar a tensão ocupacional (Amorim Neto, 2015; Simplício \& Andrade, 2011). Considerando, ainda, o comportamento do professor como modelo diretamente influenciador do comportamento e aprendizagem dos alunos, estabelece-se um ciclo crescente influenciador do ambiente educacional (Lipp, 2002; Naghieh \& cols., 2015).

Da caracterização da amostra, a porcentagem de professores do sexo feminino encontrada foi condizente com dados da literatura para pesquisas com professores de escolas estaduais, sendo a maioria casada, a maior parte com filhos; porém, em tempo de docência, nesta amostra, um pouco superior à da encontrada na literatura (Caye \& cols., 2014; Damásio \& cols., 2013; Tabeleão \& cols., 2011).

De acordo com a fase de estresse identificada, a maioria dos professores em fase de resistência, observa-se a necessidade de programas interventivos a fim de serem possibilitados comportamentos saudáveis de enfrentamento, favorecendo a promoção de resiliência e prevenindo-se patologias mais graves (Gebel, 2012).

O resultado significativo obtido referente à alteração dos sintomas de estresse (ISSL) após as intervenções corrobora Wolpe (1984) a indicar o Relaxamento Muscular como uma das formas mais eficazes de que se dispõe para redução do estresse e retoma os resultados obtidos por Esgalha (2010), que verificou alteração estatisticamente significativa para medida de estresse. Notou-se, para a EVENT, maior redução das médias de estresse do $\mathrm{G} 1$ em comparação com as médias do $\mathrm{G} 2$ e G3, e apesar de não ter sido estatisti- 
camente significativa, verificou-se tendência na redução do estresse percebido.

O fato de ter havido alteração significativa para a medida do ISSL e não para a medida de percepção de estresse da EVENT corroboram Sisto e cols.(2007) e Miguel e Noronha (2007) ao considerarem que, enquanto o ISSL mede os sintomas associados ao quadro de estresse, a EVENT mensura a vulnerabilidade da pessoa frente aos estressores encontrados no ambiente de trabalho, sendo medidas próximas, mas diferentes. De acordo com esses dados, pode-se aventar a possibilidade de que, neste período de tempo, os participantes passaram a treinar a modificação de suas respostas musculares frente aos estímulos aversivos do cotidiano, reduzindo, consequentemente, sintomas de estresse percebidos; porém, não em tempo suficiente para alterarem o incômodo frente às situações aversivas encontradas no cotidiano escolar, tais como ambiente físico inadequado e dificuldades com a chefia.

O número de professores com graus de ansiedade moderada a grave aproxima-se aos dados de Silveira e cols. (2014). Não foi obtida alteração estatisticamente significativa na medida de ansiedade após o uso da técnica, corroborando os dados encontrados no trabalho de Esgalha (2010) e de Lopes e cols. (2008). Resgatando-se os conceitos sobre o construto em questão (Barlow \& Durand, 2008), deve-se considerar a existência de variáveis não controladas, nesse experimento, no dia a dia dos professores. Levando-se em conta o fator aprendizagem, não foi possível verificar a utilização, pelos participantes, da técnica fora das sessões da pesquisa. Sublinha-se, a hipótese, portanto, de que o fator tempo de treino possa ter sido limitador do alcance da proposta frente às respostas de ansiedade como também não ter buscado informações sobre estímulos ansiogênicos e respostas apresentadas pelos participantes em seu cotidiano.

Para avaliação da QV, os dados foram condizentes com os da literatura quanto ao valor médio total obtido pelos professores de escolas públicas, bem como quanto à identificação do domínio Ambiente como o de menor avaliação (Colares \& cols., 2015); entretanto, diferiu no domínio com melhor média, identificado na literatura como o de Relações Sociais (Tabeleão \& cols., 2011; Damásio\& cols., 2013) e no presente trabalho, como o Domínio Físico. Caye e cols. (2014) obtiveram médias superiores a 70, indicando melhor percepção da QV naquela amostra, entretanto, igualando-se na pior avaliação para o Ambiente. Notou-se melhora na percepção da QV do G1 frente aos quatro domínios propostos pelo instrumento, principalmente frente ao domínio Psicológico, porém, essas alterações não foram estatisticamente significativas.

Recobrando-se a importância do uso recorrente da técnica e da insistência reiterada na prática do controle da tensão (Chep, 2013; Jacobson, 1976; Wolpe, 1984), as alterações obtidas nas medidas obtidas pela EVENT, WHOQOL e BAI podem sugerir que o número reduzido de sessões para o treino da técnica favoreceu a redução significativa dos sintomas de estresse, entretanto, não chegou a alterar significativamente o incômodo percebido pelos professores frente aos estressores ocupacionais, bem como à percepção da QV. As respostas de ansiedade, por ocorrerem frente a estímulos aversivos aprendidos, podem demandar trabalho mais focalizado e específico, com controle diferenciado para mensuração dos efeitos obtidos pelo relaxamento.

Outra hipótese a ser considerada sobre os dados obtidos é que, nesse estudo, não houve controle de treino da técnica pelos participantes fora das sessões, ou seja, embora tenham sido orientados a utilizarem o relaxamento muscular fora dos encontros, eles podem não o ter exercitado diariamente. Nesse sentido, a utilização de um diário para registro dos momentos de treino fora das sessões é uma sugestão para próximas pesquisas. A utilização dos recursos aprendidos para superar desafios de forma mais saudável é um processo de aprendizagem que se desenvolve no decorrer da vida, exigindo treino, conforme as indicações do uso da técnica (Jacobson, 1976; Wolpe, 1984).

\section{Considerações Finais}

Os dados obtidos indicaram a presença de estresse na maioria dos professores participantes, conforme dados da literatura científica e indo ao encontro da demanda por estudos interventivos voltados à redução de sintomas de estresse e ansiedade nesses profissionais. Nesse sentido, o objetivo deste estudo foi o de verificar os efeitos da técnica do Relaxamento Muscular Progressivo nas medidas de estresse, ansiedade e QV de docentes da rede pública de ensino e foi verificada diminuição significativa no estresse do grupo experimental. Para medidas da percepção de vulnerabilidade ao estresse no trabalho, ansiedade e qualidade de vida, houve alterações para o grupo interventivo indicando tendência de melhora em comparação aos outros dois grupos, porém, sem significância estatística. Esse fator pode dever-se ao curto tempo interventivo para treino da técnica.

Os estudos encontrados com propostas interventivas a fim de proporcionar melhora da saúde de docentes são reduzidos e, levando-se em conta o rigor metodológico, não foram encontradas outras pesquisas interventivas com este público considerando o tamanho amostral e delineamento quase-experimental para três grupos. Com relação à adaptação da técnica de Jacobson utilizada, a de Sandor, não foi encontrada como proposta interventiva frente a docentes e, neste ponto, verifica-se um aspecto diferente para esta pesquisa.

Ressalta-se que o assunto não se esgotou neste estudo e, resgatando as palavras de Jacobson (1976) sobre a importância da manutenção do treino do relaxamento em longo prazo a fim de se trocarem os condicionamentos adquiridos (de tensão para relaxação), surge a possibilidade de que sessões mais numerosas para treino possam favorecer o uso da técnica. Nesse sentido, verifica-se que o curto período interventivo pode ser um entrave a mudanças significativas. Deve-se levar em consideração, ainda, o fato de que as intervenções foram aplicadas em grupo, não pos- 
sibilitando um acompanhamento individual passo a passo da adaptabilidade e aprendizado da técnica.

Sugere-se que sejam feitas outras investigações de caráter interventivo com docentes, focando-se a ampliação do repertório comportamental de respostas saudáveis frente a estímulos ambientais aversivos. Ressalta-se a importância do rigor metodológico a ser proposto a fim de enriquecer o campo com pesquisas clínicas aplicadas com delineamento quase-experimental.

\section{Referências}

Almeida, L. N. A.; Lopes, L. W.; Costa, D. B.; Silva, E. G.; Cunha, G. M. S.; Almeida, A. A. F. (2014). Características vocais e emocionais de professores e não professores com baixa e alta ansiedade. Audiology - Communication Research, 19(2), 179-85. http://dx.doi. org/10.1590/S2317-64312014000200013

Amorim Neto, R. C. (2015). Teachers feel lonely too: a study of teacher's personal and professional characteristics associated with loneliness. Psico, 46(3), 321-330. http://dx.doi.org/10.15448/19808623

Andrade, P. S.; Cardoso, T. A. O. (2012). Prazer e dor na docência: revisão bibliográfica sobre a Síndrome de Burnout. Saúde e Sociedade, 21(1) 129-140. http://dx.doi.org/10.1590/S010412902012000100013.

Apóstolo, J. L. A.; Figueiredo, M. H.; Mendes, A. C.; Rodrigues, M. A. (2011). Depression, anxiety and stress in primary health care users. Revista Latino-Americana de Enfermagem, 19(2),348-353. http://dx.doi.org/10.1590/S0104-11692011000200017

Barlow, D. H.; Durand, V. M. (2008). Psicopatologia: Uma abordagem integrada (4a. ed.).São Paulo: Cengage Learning.

Brum, L. M.; Azambuja, C. R.; Rezer, J. F. P.; Temp, D. S.; Carpilovsky, C. K.; Lopes, L. F.; Schetinger, M. R. C. (2012). Qualidade de vida dos professores da área de ciências em escola pública no Rio Grande do Sul. Trabalho, Educação e Saúde, 10(1), 125-145.

Carlotto, M. S.; Dias, S. R. S.; Batista, J. B. V.; Diehl, L. (2015). O papel mediador da autoeficácia na relação entre a sobrecarga de trabalho e as dimensões de Burnout em professores. Psico-USF, 20(1), 13-23. http://dx.doi.org/10.1590/1413-82712015200102

Caye, I. T.; Ulbricht, L.; Neves, E. B. (2014). Qualidade de Vida no Trabalho dos Professores de Matemática e Português do Ensino Fundamental. Revista da Universidade Vale do Rio Verde, Três Corações, 12(2), 974-989. http://dx.doi.org/10.5892/ruvrd. v12i2.1809

Chachamovich, E.; Fleck, M. P. A. (2008). Desenvolvimento do WHOQOL-BREF. In: Fleck, M. P. A. (Org.), A avaliação de Qualidade de Vida: guia para profissionais de saúde (pp. 74-82). Porto Alegre: Artmed.
Chep, B. M. (2013). Burnout/stress management: How to reduce burnout and stress in the workplace. Journal of Healthcare Protection Management. 29(1), 92-95.

Colares, M. C.; Lopes, A. S.; Barbosa Filho, C. V.; Bavaresco, A.; Scherer, F. C. (2015). Indicadores de Qualidade de Vida em Professores: uma revisão sistemática de estudos descritivos. Pensar a Prática, 18(3), 687-708. https://doi.org/10.5216/rpp. v18i3.32128

Cunha, J. A. (2001). Manual da versão em português das Escalas Beck. São Paulo: Casa do Psicólogo.

Damásio, B. F.; Melo, R. L. P.; Silva, J. P. (2013). Sentido de Vida, Bem-Estar Psicológico e Qualidade de Vida em Professores Escolares. Paidéia, 23(54), 73-82. http://dx.doi.org/10.1590/198243272354201309

DeSousa, D. A.; Moreno, A. L.; Gauer, G.; Manfro, G. G.; Koller, S. H. (2013). Revisão sistemática de instrumentos para avaliação de ansiedade na população brasileira. Avaliação Psicológica, 12(3), $397-410$

Esgalha, L. R. (2010). Disfunção Temporomandibular (DTM): impacto do relaxamento sobre ansiedade e stress. Dissertação de Mestrado em Psicologia, Universidade Estadual Paulista Júlio de Mesquita Filho, São Paulo, SP.

Fleck, M. P.; Louzada, S.; Xavier, M.; Chachamovich, E.; Vieira, G.; Santos, L.; Pinzon, V. (2000). Aplicação da versão em português do instrumento abreviado de avaliação da Qualidade de Vida "WHOQOL-bref". Revista de Saúde Pública, 34(2),178-183. http:// dx.doi.org/10.1590/S0034-89102000000200012

Gebel, E. (2012). Getting Past Tense: dealing with stress the right way may safeguard your health. Diabetes Forecast, 65(12), 52-55. PMid:23270279.

Jacobson, E. (1976). You must to relax ( $5^{\text {th }}$ ed.). Unwin Paperbacks.

Lipp, M. E. N. (1984). Stress e suas implicações. Estudos de Psicologia, Campinas, 1 (3 e 4), 5-19.

Lipp, M. E. N. (2000). Inventário de sintomas do stress para adultos. São Paulo: Casa do Psicólogo.

Lipp, M. E. N. (Org.) (2002). O stress do professor. São Paulo: Papirus.

Lipp, M. E. N.; Malagris, L. E. N. (2001). O Stress emocional e seu tratamento In: Rangé, B. (Org.), Psicoterapias CognitivoComportamentais: um diálogo com a psiquiatria (475-490). Rio de Janeiro: Artes Médicas.

Lopes, R. F. F.; Santos, M. R.; Lopes, E. J. (2008). Efeitos do relaxamento sobre a ansiedade e desesperança em mulheres com câncer. Revista Brasileira de Terapia Comportamental Cognitiva, 
10(1), 39-49.

Miguel, F. K.; Noronha, A. P. P. (2007). Estudo dos Parâmetros Psicométricos da Escala de Vulnerabilidade ao Estresse no Trabalho. Revista Evaluar, (7), 1-18.

Moy, F. M.; Hoe, V. C. W.; Hairi, N. N.; Buckley, B.; Wark, P. A.; Koh, D.; Bueno-de-Mesquita, H. B.; Bulgiba, A. M. (2014). Cohort study on clustering of lifestyle risk factors and understanding its association with stress on health and wellbeing among school teachers in Malaysia (CLUSTer) - a study protocol. BMC Public Health, 14. Doi: 10.1186/1471-2458-14-611.

Naghieh, A.; Montgomery, P.; Bonell, C. P.; Thompson, M.; Aber, J. L. (2015). Organizational interventions for improving wellbeing and reducing work-related stress in teachers. Cochrane Database of Systematic Reviews, (4): CD010306. Doi: 10.1002/14651858. CD010306.pub2

Sandor, P. (1974). Técnicas de Relaxamento. São Paulo: Editora Vetor.

Selye, H. (1950). Stress and the General Adaptation Syndrome. British Medical Journal, 1(4667), 1383-1392. Recuperado: 15 out. 2015. Disponível: http://www.ncbi.nlm.nih.gov/pmc/articles/ PMC2038162/

Silveira, K. A.; Enumo, S. R. F.; Batista, E. P. (2014). Indicadores de estresse e estratégias de enfrentamento em professores de ensino multisseriado. Psicologia Escolar e Educacional, 18(3), 457-65.

Simplício, S. D.; Andrade, M. S. (2011). Compreendendo a questão da saúde dos professores da Rede Pública Municipal de São Paulo. Psico, 42(2), 159-167.

Sisto, F. F.; Noronha, A. P. P.; Santos, A. A. A.; Baptista, M. N. (2007). Escala de Vulnerabilidade ao Estresse no Trabalho-EVENT. São Paulo: Vetor.

Tabeleão, V. P.; Tomasi, E.; Neves, S. F. (2011). Qualidade de vida e esgotamento profissional entre docentes da rede pública de Ensino Médio e Fundamental no Sul do Brasil. Cadernos de Saúde Pública, 12(27), 2401-2408. http://dx.doi.org/10.1590/ S0102-311X2011001200011

Vale, S. F.; Maciel, R. H.; Carlotto, M. S. (2015). Propriedades psicométricas da escala de percepção de estressores ocupacionais dos professores (EPEOP). Psicologia Escolar e Educacional, 19(3), 575-583.

Wolpe, J. (1984). Deconditioning and ad hoc uses of relaxation: an overview. Journal of Behavior Therapy and Experimental Psychiatry, 15(4), 299-304. http://dx.doi.org/10.1016/00057916(84)90092-2

Recebido em: 06 de abril de 2017 Aceito em: 05 de setembro de 2017 unrestricted use,distribution and reproduction in any medium, provided the original article is properly cited. 